\title{
Projection of Machine Usage in Agriculture of Ardahan Province
}

\author{
Hasan Ertop ${ }^{1 *}$, Atılgan Atılgan ${ }^{2}$, Osman Gökdoğan $^{3}$, Burak Saltuk ${ }^{4}$ \\ ${ }^{1}$ Isparta University of Applied Sciences, Agriculture Faculty, Department of Agricultural Structures and Irrigation, Isparta/Turkey \\ (ORCID: 0000-0003-0987-5885), hasanertop@hotmail.com \\ ${ }^{2}$ Alanya Alaaddin Keykubat University, Rafet Kayıs Engineering Faculty, Department of Biosystems Engineering, Alanya-Antalya/Turkey (ORCID:0000-0003-2391- \\ 0317) atilgan.atilgan@alanya.edu.tr \\ ${ }^{3}$ Isparta University of Applied Sciences, Agriculture Faculty, Department of Agriculture Agricultural Machinery and Technologies Engineering, Isparta/Turkey \\ (ORCID: 0000-0002-4933-7144) osmangokdogan@gmail.com \\ ${ }^{4}$ Alanya Alaaddin Keykubat University Rafet Kayıs Engineering Faculty Department of Biosystems Engineering Alanya-Antalya/Turkey \\ (ORCID: 0000-0001-8673-9372) burak.saltuk@alanya.edu.tr
}

(First received 2 July 2021 and in final form 9 September 2021)

(DOI: $10.31590 /$ ejosat.961147)

ATIF/REFERENCE: Ertop, H., Atılgan, A., Gökdoğan, O., Saltuk, B., (2021). Projection of Machine Usage in Agriculture of Ardahan Province. European Journal of Science and Technology, (27), 259-266.

\begin{abstract}
In this study, the usage projection of agricultural machinery used in agricultural operations in Ardahan province was examined. It is aimed to determine the projection of technology use in agriculture by using the data between 2011-2020. The change rates of 35 machines, including tillage, sowing, planting, fertilization, spraying, harvesting-threshing and other machines, between 2011-2020 were examined. Projection coefficients were calculated and by using these, coefficients were calculated and evaluated for the years 2021 2030. In the study, the data of the Turkish Statistical Institute and findings from the related studies on the subject were used. It has been concluded that the positive projection coefficients of 29 machines considered in the technology usage projection will increase until 2030, while the projection coefficient determined for 6 machines is negative and there will be a decrease in these machines.
\end{abstract}

Keywords: Ardahan, Projection, Agricultural machinery

\section{Ardahan İli Tarımda Makine Kullanımı Projeksiyonu}

$\ddot{O} \mathbf{z}$

Bu çalışmada Ardahan ilinde tarımsal işlemlerde kullanılan tarım makinelerinin kullanım projeksiyonu incelenmiştir. 2011-2020 yılları arasındaki veriler kullanılarak tarımda teknoloji kullanım projeksiyonunun belirlenmesi hedeflenmiştir. 2011-2020 yılları arasında toprak işleme, ekim, dikim, gübreleme, ilaçlama, hasat-harman ve diğer makineleri içeren 35 makinenin değişim oranları incelenmiştir. Projeksiyon katsayıları hesaplanmış ve bunlar kullanılarak 2021-2030 yılları için katsayılar hesaplanmış ve değerlendirilmiştir. Çalışmada, Türkiye İstatistik Kurumu'nun verileri ve konu ile ilgili yapılan çalışmalardan elde edilen bulgular kullanılmıştır. Teknoloji kullanım projeksiyonunda ele alınan 29 makinenin pozitif projeksiyon katsayılarının 2030 yılına kadar artacağı, 6 makine için belirlenen projeksiyon katsayısının ise negatif olduğu ve bu makinelerde azalma olacağı sonucuna varılmıştır.

Anahtar Kelimeler: Ardahan, Projeksiyon, Tarım makineleri.

\footnotetext{
* Corresponding author: Alanya Alaaddin Keykubat University Rafet Kayıs Engineering Faculty Department of Biosystems Engineering AlanyaAntalya/Turkey (ORCID: 0000-0001-8673-9372) burak.saltuk@alanya.edu.tr
} 


\section{Introduction}

Mechanization has great importance in increasing production, work efficiency and quality of work in agriculture, facilitating work, reducing costs, modernizing enterprises, opening new business areas, and improving the socio-economical population of agriculture. (Altay and Turhal, 2011). In addition to the necessity of using natural resources effectively and efficiently for the sustainability of agricultural production, it is obligatory to apply techniques that can help the people in production to develop socio-economically. In order to fulfil these conditions, it is necessary to eliminate the deficiencies of the implemented system and to develop new and effective technologies (Kaya et al., 2010).

Agricultural mechanization shows different levels of development and practice in all countries. This difference can be seen in the regions of a country as well as in agricultural enterprises within the same region. Depending on the technical and economic structure of the agricultural enterprise, the level of agricultural mechanization can be in different values (Koçtürk and Onurbaş Avc1oğlu, 2007). Conducting studies in the field of agricultural mechanization with up-to-date data in line with developments in agricultural production systems and technologies; It is of great importance for making the right decisions for the future by contributing to the agricultural development plans at regional, national and provincial level (Bayram and Altuntaş, 2016).

The use of machinery in agriculture, unlike other applications of agricultural technology, indirectly affects the increase in productivity and provides the application of new production methods in rural areas. In this respect, it increases the efficiency and economy of other technological applications and improves working conditions. Thus, by enabling the use of appropriate technologies, it helps to get more efficiency from production areas of a certain size (Saral et al., 2000; Demir and Kuş, 2016). In this study, it is aimed to determine the agricultural mechanization projection of the province of Ardahan for the next ten years, until 2030 and these values will guide the mechanization plans in the region.

\section{Material and Method}

Located in the northeast of Anatolia, Ardahan Province is surrounded by Georgia and Armenia in the northeast, Kars in the south and southeast, Erzurum in the southwest and Artvin in the west. Due to the high altitude of the region and the variability of the surface shapes, the continental climate prevails throughout the province, and the winters are long, harsh and snowy. In the province, which has an altitude of $1829 \mathrm{~m}$ above sea level, it is observed that the temperatures can reach the highest point at 33.6 ${ }^{\circ} \mathrm{C}$ in summer and decrease to $-30.4{ }^{\circ} \mathrm{C}$ in winter (Anonymous, 2021a). The agricultural area of Ardahan province yields cereals and other herbal products on 427392 da, vegetables on 90 decares, and fruits, beverage and spice crops on 502 decares. The total agricultural area of Ardahan province is 427984 da, excluding meadow and pasture areas (Anonymous, 2021b). The material of the study consisted of the agricultural machinery data of the Turkish Statistical Institute for the years 2011-2020 for the province of Ardahan in general (Anonymous, 2021c). The projection coefficient was calculated based on the production and usage amounts of the technology used in agriculture in the past ten years. In this context, the usage projection of 35 machines commonly used in Ardahan (tillage, sowing, planting, fertilization, spraying, harvesting-threshing and other machines) has been taken into consideration.

In line with the increase or decrease in the projection coefficient, ten-year projections of technology-use in agriculture in Ardahan province were determined. Depending on the number of machines in the previous year and the coefficient determined for that machine, the projections up to year 2030 of the agricultural machines widely used in Ardahan were determined by using the method of Demir and Kuş (2016), Baran et al. (2019), Solak et al. (2019). A positive projection coefficient indicates an increase in the number of existing machines, and a negative projection indicates a decrease (Demir, 2013; Demir and Kuş, 2016).

\section{Results and Discussion}

In the study, the number of soil tillage machines in the past ten years, the change rates compared to previous years, the projection coefficients calculated depending on the number of machines and the number of machines in the ten-year period are given in Table 1. According to the change rates calculated for the years 2011-2020, as seen in Table 1, the projection coefficients for the mouldboard plow, tooth harrow, disc harrow, cultivator, disc plow, mouldboard stubble plow, roller, arc opening plow, disc stubble plow and rotary tiller are $-0.06,0.67,-0.51,38.40,17.22$, $4.27,-12.61,3.30,9.17$ and $13.86(\%)$ respectively. According to the projection coefficient calculated for the years 2021 and 2030, it has been calculated that the largest increase will be observed in cultivator by $38.40 \%$ and corresponding to 10572 units, and the largest decrease will be observed in roller by -12.61 and corresponding to 18 units. The importance attached to tillage before sowing in grain production can explain the high number of mouldboard tractor plows.

The number of machines for sowing, planting and fertilizing machines in the past ten years, the rate of change compared to previous years, the projection coefficients calculated depending on the number of machines and the number of machines in the ten-year period are given in Table 2. According to the change rates calculated for the years 2011-2020, as seen in Table 2, the projection coefficients calculated for the chemical fertilizer spreader, tractor seed drill, combined seed drill, solid manure spreader and potato planting machine are 4.51, 2.83, 9.04, 37.16 and $25.93(\%)$ respectively. According to the projection coefficient calculated for the years 2021 and 2030, it has been calculated that the biggest increase will be in the solid manure spreader by $37.16 \%$ corresponding to 10572 units. It is possible to claim that the increase in the number of combined grain seeding machines over the years in Ardahan is beauce of the importance of grain production. Similarly, the number of potato planting machines increased from 1 unit in 2011 to 4 units in 2020. With the projection coefficient of $25.93 \%$ stated in Table 2, it can be assumed that the number of potato planting machines will increase to 40 in 2030. It can be thought that the low number of potato planting machines is due to the fact that potato cultivation is limited to an area of $292.48 \mathrm{da}$ (Anonymous, 2021d) according to 2020 data and grain production has a greater importance in provincial agriculture.

The number of machines belonging to plant protection machinery in the past ten years, the rate of change compared to 
previous years, the projection coefficients calculated depending on the number of machines and the number of machines in the ten-year period are given in Table 3. According to Table 3, indicating the change rates for the 2011-2020 period, the projection coefficients for back sprayer; stretcher, motorized sprayer duster combined atomizer; motorized sprayer and atomizer have been respectively calculated as $2.35,6.79,-0.13$ and $5.40(\%)$. According to the projection coefficient calculated for the 2021-2030 period, the greatest increase will be in in stretcher, motorized sprayer duster combined atomizer by $6.79 \%$, corresponding to 23 units, and the greatest reduction will be observed in motorised sprayer by $-0.13 \%$, corresponding to 9 units. As indicated in Table 3, it can be said that there is not much tendency towards spraying machines in the province and therefore, plant protection activities with pesticide coverage are not carried out too much. According to Anonymous (2021a), this can be explained by the fact that almost all of the plant production and bee products in the province are ecological.

The number of harvesting-threshing machines in the past ten years, the change rates compared to previous years, the projection coefficients calculated depending on the number of machines and the number of machines in the ten-year period are given in Table 4. The projection coefficients calculated for the harvester and threshing machine, tractor pulled mower, hay rake, straw transferunloading machine, reaper, hay making machine, hay silage machine, corn silage machine and potato harvesting machine were calculated as $0.24,5.99,2.89,15.94,6.51,-8.89,6.15,5.56,5.56$ and 20.37 respectively (Table 4). According to the projection coefficient calculated for the years 2021 and 2030, it has been calculated that the biggest increase will be in the potato harvester by $38.40 \%$ corresponding to 26 pieces and the biggest decrease will be observed in the number of reapers by -8.89 corresponding to 20 units. The fact that grain production has a greater importance in provincial agriculture can possibly have an effect on this. The high number of harvesting and thresher machines can indicate that grain production has a greater importance in provincial agriculture.

The number of other agricultural machinery for the past decade, the rate of change compared to previous years, projection coefficients and the number of machinery in the ten-year period are given in Table 5. The projection coefficients calculated for cream machine, churn, milking machine (mobile), milking facility, incubator, trailer, ladle, feed preparation machine, water tank and pump are $-0.84,0.08,22.62,37.62,42.40,2.18,8.43$, $1.19,17.88$ and $1.30(\%)$ respectively (Table 5). According to the projection coefficient calculated for 2021 and 2030, it has been calculated that the biggest increase will be observed in incubator by $42.40 \%$ corresponding to 823 units, and the biggest decrease will be observed in cream machines by -0.84 corresponding to 8611 units. This can be explained by the fact that the economy of the province is based on agriculture in general and livestock sector in particular (Anonymous, 2021a).

Table 1. Soil tillage machinery projection

\begin{tabular}{|c|c|c|c|c|c|c|c|c|c|c|}
\hline Past Years & \begin{tabular}{|c|} 
Mouldboard \\
Plow
\end{tabular} & $\begin{array}{c}\text { Tooth } \\
\text { Harrow }\end{array}$ & $\begin{array}{c}\text { Disc } \\
\text { Harrow }\end{array}$ & $\begin{array}{c}\text { Cultiv } \\
\text { ator }\end{array}$ & $\begin{array}{l}\text { Disc } \\
\text { Plow }\end{array}$ & $\begin{array}{c}\text { Mouldb } \\
\text { oard } \\
\text { Stubble } \\
\text { Plow } \\
\end{array}$ & \begin{tabular}{|c|} 
Rolle \\
$\mathbf{r}$
\end{tabular} & $\begin{array}{c}\text { Arc Opening } \\
\text { Plow } \\
\end{array}$ & $\begin{array}{c}\text { Disc Stubble } \\
\text { Plow } \\
\end{array}$ & $\begin{array}{c}\text { Rotary } \\
\text { Tiller }\end{array}$ \\
\hline 2011 & 5670 & 3092 & 1141 & 137 & 47 & 114 & 524 & 52 & 15 & 5 \\
\hline 2012 & 5790 & 3152 & 1141 & 145 & 47 & 109 & 304 & 52 & 16 & 6 \\
\hline 2013 & 5810 & 3177 & 1146 & 149 & 48 & 109 & 304 & 52 & 16 & 6 \\
\hline 2014 & 5600 & 3181 & 1150 & 149 & 49 & 109 & 304 & 53 & 16 & 6 \\
\hline 2015 & 5719 & 3207 & 1158 & 150 & 59 & 118 & 311 & 52 & 17 & 8 \\
\hline 2016 & 5750 & 3234 & 1160 & 153 & 109 & 116 & 319 & 53 & 17 & 8 \\
\hline 2017 & 5470 & 3384 & 1075 & 732 & 144 & 117 & 326 & 60 & 18 & 10 \\
\hline 2018 & 5484 & 3384 & 1076 & 732 & 144 & 118 & 326 & 60 & 19 & 10 \\
\hline 2019 & 5569 & 3221 & 1076 & 411 & 158 & 122 & 327 & 66 & 21 & 11 \\
\hline 2020 & 5625 & 3275 & 1087 & 410 & 164 & 160 & 69 & 69 & 31 & 15 \\
\hline $\begin{array}{c}\text { Exchange } \\
\text { Rates }\end{array}$ & \begin{tabular}{|c|} 
Mouldboard \\
Plow
\end{tabular} & $\begin{array}{c}\text { Tooth } \\
\text { Harrow }\end{array}$ & $\begin{array}{c}\text { Disc } \\
\text { Harrow }\end{array}$ & $\begin{array}{l}\text { Cultiv } \\
\text { ator }\end{array}$ & $\begin{array}{l}\text { Disc } \\
\text { Plow }\end{array}$ & $\begin{array}{c}\text { Mouldb } \\
\text { oard } \\
\text { Stubble } \\
\text { Plow } \\
\end{array}$ & \begin{tabular}{|c|} 
Rolle \\
$\mathbf{r}$
\end{tabular} & $\begin{array}{c}\text { Arc Opening } \\
\text { Plow } \\
\end{array}$ & $\begin{array}{c}\text { Disc Stubble } \\
\text { Plow }\end{array}$ & $\begin{array}{c}\text { Rotary } \\
\text { Tiller }\end{array}$ \\
\hline 2011-2012 & 2.12 & 1.94 & 0.00 & 5.84 & 0.00 & -4.39 & -41.98 & 0.00 & 6.67 & 20.00 \\
\hline 2012-2013 & 0.35 & 0.79 & 0.44 & 2.76 & 2.13 & 0.00 & 0.00 & 0.00 & 0.00 & 0.00 \\
\hline 2013-2014 & -3.61 & 0.13 & 0.35 & 0.00 & 2.08 & 0.00 & 0.00 & 1.92 & 0.00 & 0.00 \\
\hline 2014-2015 & 2.13 & 0.82 & 0.70 & 0.67 & 20.41 & 8.26 & 2.30 & -1.89 & 6.25 & 33.33 \\
\hline $2015-2016$ & 0.54 & 0.84 & 0.17 & 2.00 & 84.75 & -1.69 & 2.57 & 1.92 & 0.00 & 0.00 \\
\hline 2016-2017 & -4.87 & 4.64 & -7.33 & 378.43 & 32.11 & 0.86 & 2.19 & 13.21 & 5.88 & 25.00 \\
\hline $2017-2018$ & 0.26 & 0.00 & 0.09 & 0.00 & 0.00 & 0.85 & 0.00 & 0.00 & 5.56 & 0.00 \\
\hline 2018-2019 & 1.55 & -4.82 & 0.00 & -43.85 & 9.72 & 3.39 & 0.31 & 10.00 & 10.53 & 10.00 \\
\hline 2019-2020 & 1.01 & 1.68 & 1.02 & -0.24 & 3.80 & 31.15 & -78.90 & 4.55 & 47.62 & 36.36 \\
\hline$\%$ & -0.06 & 0.67 & -0.51 & 38.40 & 17.22 & 4.27 & \begin{tabular}{|l|}
-12.61 \\
\end{tabular} & 3.30 & 9.17 & 13.86 \\
\hline
\end{tabular}




\begin{tabular}{|c|c|c|c|c|c|c|c|c|c|c|}
\hline Projection & $\begin{array}{c}\text { Mouldboard } \\
\text { Plow }\end{array}$ & $\begin{array}{c}\text { Tooth } \\
\text { Harrow }\end{array}$ & $\begin{array}{c}\text { Disc } \\
\text { Harrow }\end{array}$ & $\begin{array}{c}\text { Cultiv } \\
\text { ator }\end{array}$ & $\begin{array}{c}\text { Disc } \\
\text { Plow }\end{array}$ & $\begin{array}{c}\text { Mould } \\
\text { board } \\
\text { Stubble } \\
\text { Plow }\end{array}$ & Roller & $\begin{array}{c}\text { Arc Opening } \\
\text { Plow }\end{array}$ & $\begin{array}{c}\text { Disc Stubble } \\
\text { Plow }\end{array}$ & $\begin{array}{c}\text { Rotary } \\
\text { Tiller }\end{array}$ \\
\hline $\mathbf{2 0 2 1}$ & 5622 & 3297 & 1081 & 567 & 192 & 167 & 60 & 71 & 34 & 17 \\
\hline $\mathbf{2 0 2 2}$ & 5618 & 3319 & 1076 & 785 & 225 & 174 & 53 & 74 & 37 & 19 \\
\hline $\mathbf{2 0 2 3}$ & 5615 & 3341 & 1070 & 1087 & 264 & 181 & 46 & 76 & 40 & 22 \\
\hline $\mathbf{2 0 2 4}$ & 5612 & 3364 & 1065 & 1504 & 310 & 189 & 40 & 79 & 44 & 25 \\
\hline $\mathbf{2 0 2 5}$ & 5608 & 3386 & 1060 & 2082 & 363 & 197 & 35 & 81 & 48 & 29 \\
\hline $\mathbf{2 0 2 6}$ & 5605 & 3409 & 1054 & 2881 & 425 & 206 & 31 & 84 & 52 & 33 \\
\hline $\mathbf{2 0 2 7}$ & 5601 & 3432 & 1049 & 3988 & 499 & 214 & 27 & 87 & 57 & 37 \\
\hline $\mathbf{2 0 2 8}$ & 5598 & 3455 & 1043 & 5519 & 585 & 224 & 23 & 89 & 63 & 42 \\
\hline $\mathbf{2 0 2 9}$ & 5595 & 3478 & 1038 & 7639 & 685 & 233 & 21 & 92 & 68 & 48 \\
\hline $\mathbf{2 0 3 0}$ & 5591 & 3501 & 1033 & 10572 & 803 & 243 & 18 & 95 & 75 & 55 \\
\hline
\end{tabular}

Table 2. Sowing, planting and fertilizing machinery projection

\begin{tabular}{|c|c|c|c|c|c|}
\hline Past Years & $\begin{array}{c}\text { Chemical Fertilizer } \\
\text { Spreader }\end{array}$ & $\begin{array}{c}\text { Tractor Seed } \\
\text { Drill }\end{array}$ & $\begin{array}{c}\text { Combined Seed } \\
\text { Drill }\end{array}$ & $\begin{array}{c}\text { Solid Manure } \\
\text { Spreader }\end{array}$ & $\begin{array}{c}\text { Potato Planting } \\
\text { Machine }\end{array}$ \\
\hline 2011 & 327 & 43 & 25 & 2 & 1 \\
\hline 2012 & 355 & 45 & 35 & 3 & 1 \\
\hline 2013 & 393 & 48 & 40 & 3 & 1 \\
\hline 2014 & 408 & 49 & 42 & 3 & 1 \\
\hline 2015 & 407 & 48 & 43 & 10 & 3 \\
\hline 2016 & 423 & 48 & 45 & 11 & 3 \\
\hline 2017 & 450 & 49 & 45 & 14 & 4 \\
\hline 2018 & 462 & 49 & 45 & 14 & 4 \\
\hline 2019 & 482 & 50 & 48 & 15 & 4 \\
\hline 2020 & 484 & 55 & 52 & 16 & 4 \\
\hline $\begin{array}{c}\text { Exchange } \\
\text { Rates }\end{array}$ & $\begin{array}{c}\text { Chemical Fertilizer } \\
\text { Spreader }\end{array}$ & $\begin{array}{c}\text { Tractor Seed } \\
\text { Drill }\end{array}$ & $\begin{array}{c}\text { Combined Seed } \\
\text { Drill }\end{array}$ & $\begin{array}{c}\text { Solid Manure } \\
\text { Spreader }\end{array}$ & $\begin{array}{c}\text { Potato Planting } \\
\text { Machine }\end{array}$ \\
\hline 2011-2012 & 8.56 & 4.65 & 40.00 & 50.00 & 0.00 \\
\hline $2012-2013$ & 10.70 & 6.67 & 14.29 & 0.00 & 0.00 \\
\hline 2013-2014 & 3.82 & 2.08 & 5.00 & 0.00 & 0.00 \\
\hline 2014-2015 & -0.25 & -2.04 & 2.38 & 233.33 & 200.00 \\
\hline 2015-2016 & 3.93 & 0.00 & 4.65 & 10.00 & 0.00 \\
\hline 2016-2017 & 6.38 & 2.08 & 0.00 & 27.27 & 33.33 \\
\hline $2017-2018$ & 2.67 & 0.00 & 0.00 & 0.00 & 0.00 \\
\hline 2018-2019 & 4.33 & 2.04 & 6.67 & 7.14 & 0.00 \\
\hline 2019-2020 & 0.41 & 10.00 & 8.33 & 6.67 & 0.00 \\
\hline$\%$ & 4.51 & 2.83 & 9.04 & 37.16 & 25.93 \\
\hline Projection & $\begin{array}{c}\text { Chemical Fertilizer } \\
\text { Spreader }\end{array}$ & $\begin{array}{c}\text { Tractor Seed } \\
\text { Drill }\end{array}$ & $\begin{array}{c}\text { Combined Seed } \\
\text { Drill }\end{array}$ & $\begin{array}{c}\text { Solid Manure } \\
\text { Spreader }\end{array}$ & $\begin{array}{c}\text { Potato Planting } \\
\text { Machine }\end{array}$ \\
\hline 2021 & 506 & 57 & 57 & 22 & 5 \\
\hline 2022 & 529 & 58 & 62 & 30 & 6 \\
\hline 2023 & 552 & 60 & 67 & 41 & 8 \\
\hline 2024 & 577 & 61 & 74 & 57 & 10 \\
\hline 2025 & 603 & 63 & 80 & 78 & 13 \\
\hline 2026 & 631 & 65 & 87 & 107 & 16 \\
\hline 2027 & 659 & 67 & 95 & 146 & 20 \\
\hline 2028 & 689 & 69 & 104 & 200 & 25 \\
\hline 2029 & 720 & 71 & 113 & 275 & 32 \\
\hline 2030 & 752 & 73 & 124 & 377 & 40 \\
\hline
\end{tabular}


European Journal of Science and Technology

Table 3. Plant protection machinery projection

\begin{tabular}{|c|c|c|c|c|}
\hline $\begin{array}{l}\text { Past } \\
\text { Years }\end{array}$ & Back Sprayer & Stretcher, Motorized Sprayer Duster Combined Atomizer & Motorized Sprayer & Atomizer \\
\hline 2011 & 51 & 8 & 11 & 6 \\
\hline 2012 & 47 & 8 & 11 & 6 \\
\hline 2013 & 52 & 13 & 13 & 5 \\
\hline 2014 & 50 & 12 & 12 & 5 \\
\hline 2015 & 55 & 12 & 10 & 6 \\
\hline 2016 & 57 & 12 & 10 & 6 \\
\hline 2017 & 58 & 9 & 8 & 6 \\
\hline 2018 & 58 & 9 & 8 & 6 \\
\hline 2019 & 61 & 11 & 10 & 7 \\
\hline 2020 & 62 & 12 & 10 & 9 \\
\hline $\begin{array}{c}\text { Exchange } \\
\text { Rates } \\
\end{array}$ & Back Sprayer & Stretcher, Motorized Sprayer Duster Combined Atomizer & Motorized Sprayer & Atomizer \\
\hline 2011-2012 & -7.84 & 0.00 & 0.00 & 0.00 \\
\hline 2012-2013 & 10.64 & 62.50 & 18.18 & -16.67 \\
\hline 2013-2014 & -3.85 & -7.69 & -7.69 & 0.00 \\
\hline 2014-2015 & 10.00 & 0.00 & -16.67 & 20.00 \\
\hline 2015-2016 & 3.64 & 0.00 & 0.00 & 0.00 \\
\hline 2016-2017 & 1.75 & -25.00 & -20.00 & 0.00 \\
\hline $2017-2018$ & 0.00 & 0.00 & 0.00 & 0.00 \\
\hline 2018-2019 & 5.17 & 22.22 & 25.00 & 16.67 \\
\hline 2019-2020 & 1.64 & 9.09 & 0.00 & 28.57 \\
\hline$\%$ & 2.35 & 6.79 & -0.13 & 5.40 \\
\hline Projection & Back Sprayer & Stretcher, Motorized Sprayer Duster Combined Atomizer & Motorized Sprayer & Atomizer \\
\hline 2021 & 63 & 13 & 9 & 9 \\
\hline 2022 & 65 & 14 & 9 & 10 \\
\hline 2023 & 66 & 15 & 9 & 11 \\
\hline 2024 & 68 & 16 & 9 & 11 \\
\hline 2025 & 70 & 17 & 9 & 12 \\
\hline 2026 & 71 & 18 & 9 & 12 \\
\hline 2027 & 73 & 19 & 9 & 13 \\
\hline 2028 & 75 & 20 & 9 & 14 \\
\hline 2029 & 76 & 22 & 9 & 14 \\
\hline 2030 & 78 & 23 & 9 & 15 \\
\hline
\end{tabular}

Table 4. Harvest-threshing machinery projection

\begin{tabular}{|c|c|c|c|c|c|c|c|c|c|c|}
\hline $\begin{array}{l}\text { Past } \\
\text { Years }\end{array}$ & $\begin{array}{c}\text { Harvester and Threshing } \\
\text { Machine (Thrasher) }\end{array}$ & $\begin{array}{l}\text { Tractor } \\
\text { Pulled } \\
\text { Mower }\end{array}$ & $\begin{array}{l}\text { Hay } \\
\text { Rake }\end{array}$ & $\begin{array}{c}\text { Baler } \\
\text { Machin } \\
\text { e }\end{array}$ & $\begin{array}{c}\text { Hay Transfer- } \\
\text { Unloading Machine }\end{array}$ & $\begin{array}{l}\text { Rea } \\
\text { per }\end{array}$ & $\begin{array}{c}\text { Stra } \\
\text { w } \\
\text { Mak } \\
\text { ing } \\
\text { Mac } \\
\text { hine }\end{array}$ & $\begin{array}{c}\text { Hay } \\
\text { Silag } \\
\text { e } \\
\text { Mac } \\
\text { hine }\end{array}$ & $\begin{array}{c}\text { Cor } \\
\text { n } \\
\text { Silag } \\
\text { e } \\
\text { Mac } \\
\text { hine }\end{array}$ & $\begin{array}{c}\text { Potat } \\
\text { o } \\
\text { Harve } \\
\text { sting } \\
\text { Machi } \\
\text { ne }\end{array}$ \\
\hline 2011 & 4194 & 2075 & 2469 & 150 & 128 & 250 & 26 & 2 & 2 & 1 \\
\hline 2012 & 4185 & 2220 & 2559 & 232 & 173 & 250 & 27 & 2 & 2 & 2 \\
\hline 2013 & 4199 & 2351 & 2623 & 270 & 174 & 250 & 29 & 2 & 2 & 2 \\
\hline 2014 & 4303 & 2493 & 2638 & 318 & 176 & 250 & 30 & 2 & 2 & 2 \\
\hline 2015 & 4343 & 2548 & 2810 & 343 & 195 & 50 & 31 & 2 & 2 & 3 \\
\hline 2016 & 4381 & 2568 & 2864 & 349 & 194 & 50 & 37 & 2 & 2 & 3 \\
\hline 2017 & 4406 & 2969 & 2976 & 386 & 200 & 50 & 40 & 2 & 2 & 4 \\
\hline 2018 & 4411 & 2969 & 3146 & 392 & 200 & 50 & 40 & 2 & 2 & 4 \\
\hline 2019 & 4094 & 3102 & 3192 & 487 & 210 & 50 & 43 & 2 & 3 & 4 \\
\hline 2020 & 4268 & 3471 & 3184 & 529 & 217 & 50 & 44 & 3 & 3 & 4 \\
\hline
\end{tabular}


Avrupa Bilim ve Teknoloji Dergisi

\begin{tabular}{|c|c|c|c|c|c|c|c|c|c|c|}
\hline $\begin{array}{c}\text { Excha } \\
\text { nge } \\
\text { Rates }\end{array}$ & $\begin{array}{l}\text { Harvester and Threshing } \\
\text { Machine (Thrasher) }\end{array}$ & $\begin{array}{c}\text { Tractor } \\
\text { Pulled Mower }\end{array}$ & $\begin{array}{c}\text { Hay } \\
\text { Rake }\end{array}$ & $\begin{array}{c}\text { Baler } \\
\text { Machine }\end{array}$ & $\begin{array}{c}\text { Hay Transfer- } \\
\text { Unloading Machine }\end{array}$ & $\begin{array}{l}\text { Rea } \\
\text { per }\end{array}$ & $\begin{array}{c}\text { Stra } \\
\text { w } \\
\text { Maki } \\
\text { ng } \\
\text { Mac } \\
\text { hine }\end{array}$ & \begin{tabular}{|c|} 
Hay \\
Silag \\
e \\
Mac \\
hine
\end{tabular} & $\begin{array}{c}\text { Corn } \\
\text { Silag } \\
\text { e } \\
\text { Mac } \\
\text { hine }\end{array}$ & $\begin{array}{c}\text { Potato } \\
\text { Harves } \\
\text { ting } \\
\text { Machi } \\
\text { ne }\end{array}$ \\
\hline $\begin{array}{l}\text { 2011- } \\
2012\end{array}$ & -0.21 & 6.99 & 3.65 & 54.67 & 35.16 & 0.00 & 3.85 & 0.00 & 0.00 & 100.00 \\
\hline $\begin{array}{l}2012- \\
2013\end{array}$ & 0.33 & 5.90 & 2.50 & 16.38 & 0.58 & 0.00 & 7.41 & 0.00 & 0.00 & 0.00 \\
\hline $\begin{array}{l}2013- \\
2014 \\
\end{array}$ & 2.48 & 6.04 & 0.57 & 17.78 & 1.15 & 0.00 & 3.45 & 0.00 & 0.00 & 0.00 \\
\hline $\begin{array}{l}2014- \\
2015 \\
\end{array}$ & 0.93 & 2.21 & 6.52 & 7.86 & 10.80 & $\begin{array}{c}- \\
80.0 \\
0\end{array}$ & 3.33 & 0.00 & 0.00 & 50.00 \\
\hline $\begin{array}{l}2015- \\
2016 \\
\end{array}$ & 0.87 & 0.78 & 1.92 & 1.75 & -0.51 & 0.00 & 19.35 & 0.00 & 0.00 & 0.00 \\
\hline $\begin{array}{l}2016- \\
2017 \\
\end{array}$ & 0.57 & 15.62 & 3.91 & 10.60 & 3.09 & 0.00 & 8.11 & 0.00 & 0.00 & 33.33 \\
\hline $\begin{array}{l}2017- \\
2018 \\
\end{array}$ & 0.11 & 0.00 & 5.71 & 1.55 & 0.00 & 0.00 & 0.00 & 0.00 & 0.00 & 0.00 \\
\hline $\begin{array}{l}2018- \\
2019 \\
\end{array}$ & -7.19 & 4.48 & 1.46 & 24.23 & 5.00 & 0.00 & 7.50 & 0.00 & 50.00 & 0.00 \\
\hline $\begin{array}{l}2019- \\
2020\end{array}$ & 4.25 & 11.90 & -0.25 & 8.62 & 3.33 & 0.00 & 2.33 & 50.00 & 0.00 & 0.00 \\
\hline$\%$ & 0.24 & 5.99 & 2.89 & 15.94 & 6.51 & $\begin{array}{c}- \\
8.89 \\
\end{array}$ & 6.15 & 5.56 & 5.56 & 20.37 \\
\hline $\begin{array}{l}\text { Proje } \\
\text { ction }\end{array}$ & $\begin{array}{c}\text { Harvester and Threshing } \\
\text { Machine (Thrasher) }\end{array}$ & $\begin{array}{l}\text { Tractor } \\
\text { Pulled } \\
\text { Mower } \\
\end{array}$ & $\begin{array}{l}\text { Hay } \\
\text { Rake } \\
\end{array}$ & $\begin{array}{c}\text { Baler } \\
\text { Machin } \\
\text { e } \\
\end{array}$ & $\begin{array}{c}\text { Hay Transfer- } \\
\text { Unloading Machine } \\
\end{array}$ & $\begin{array}{l}\text { Rea } \\
\text { per }\end{array}$ & $\begin{array}{c}\text { Stra } \\
\text { w } \\
\text { Mak } \\
\text { ing } \\
\text { Mac } \\
\text { hine } \\
\end{array}$ & \begin{tabular}{|c|} 
Hay \\
Silag \\
e \\
Mac \\
hine \\
\end{tabular} & $\begin{array}{c}\text { Cor } \\
\text { n } \\
\text { Silag } \\
\text { e } \\
\text { Mac } \\
\text { hine } \\
\end{array}$ & $\begin{array}{c}\text { Potat } \\
\text { o } \\
\text { Harve } \\
\text { sting } \\
\text { Machi } \\
\text { ne } \\
\end{array}$ \\
\hline 2021 & 4278 & 5550 & 3276 & 613 & 231 & 46 & 47 & 3 & 3 & 5 \\
\hline 2022 & 4289 & 5883 & 3371 & 711 & 246 & 42 & 50 & 3 & 3 & 6 \\
\hline 2023 & 4299 & 6235 & 3468 & 824 & 262 & 38 & 53 & 4 & 4 & 7 \\
\hline 2024 & 4309 & 6608 & 3568 & 956 & 279 & 34 & 56 & 4 & 4 & 8 \\
\hline 2025 & 4319 & 7004 & 3671 & 1108 & 297 & 31 & 59 & 4 & 4 & 10 \\
\hline 2026 & 4330 & 7424 & 3778 & 1285 & 317 & 29 & 63 & 4 & 4 & 12 \\
\hline 2027 & 4340 & 7869 & 3887 & 1490 & 337 & 26 & 67 & 4 & 4 & 15 \\
\hline 2028 & 4351 & 8340 & 3999 & 1727 & 359 & 24 & 71 & 5 & 5 & 18 \\
\hline 2029 & 4361 & 8839 & 4115 & 2002 & 383 & 22 & 75 & 5 & 5 & 21 \\
\hline 2030 & 4372 & 9369 & 4234 & 2322 & 408 & 20 & 80 & 5 & 5 & 26 \\
\hline
\end{tabular}

Table 5. Projection of other machinery

\begin{tabular}{|c|c|c|c|c|c|c|c|c|c|c|}
\hline $\begin{array}{c}\text { Previo } \\
\text { us } \\
\text { Years }\end{array}$ & $\begin{array}{c}\text { Cream } \\
\text { Machine }\end{array}$ & $\begin{array}{c}\text { Chur } \\
\text { n }\end{array}$ & $\begin{array}{l}\text { Milking } \\
\text { Machine } \\
\text { (Mobile) }\end{array}$ & $\begin{array}{l}\text { Milking } \\
\text { Facility }\end{array}$ & $\begin{array}{c}\text { Incubat } \\
\text { or }\end{array}$ & $\begin{array}{c}\text { Trail } \\
\text { er }\end{array}$ & $\begin{array}{c}\text { Lad } \\
\text { le }\end{array}$ & $\begin{array}{c}\text { Feed Preparation } \\
\text { Machine }\end{array}$ & $\begin{array}{l}\text { Water } \\
\text { Tank }\end{array}$ & $\begin{array}{c}\text { Pum } \\
\text { p }\end{array}$ \\
\hline 2011 & 10069 & 6729 & 281 & 30 & 3 & 4683 & 176 & 111 & 23 & 150 \\
\hline 2012 & 10129 & 6889 & 458 & 30 & 3 & 4733 & 179 & 111 & 27 & 150 \\
\hline 2013 & 10109 & 6795 & 588 & 40 & 3 & 4808 & 179 & 113 & 29 & 152 \\
\hline 2014 & 10077 & 6345 & 713 & 53 & 3 & 4841 & 182 & 111 & 30 & 150 \\
\hline 2015 & 10134 & 6454 & 732 & 53 & 12 & 5047 & 211 & 106 & 32 & 148 \\
\hline 2016 & 10150 & 6492 & 739 & 48 & 17 & 5082 & 209 & 107 & 32 & 150 \\
\hline 2017 & 10137 & 6498 & 1316 & 53 & 22 & 5346 & 223 & 113 & 81 & 150 \\
\hline 2018 & 10222 & 6458 & 1327 & 53 & 22 & 5351 & 223 & 113 & 81 & 150 \\
\hline 2019 & 10267 & 6483 & 1349 & 56 & 26 & 5501 & 333 & 118 & 59 & 162 \\
\hline 2020 & 9291 & 6752 & 1440 & 205 & 24 & 5677 & 338 & 123 & 59 & 168 \\
\hline
\end{tabular}




\begin{tabular}{|c|c|c|c|c|c|c|c|c|c|c|}
\hline $\begin{array}{l}\text { Change } \\
\text { Rates }\end{array}$ & $\begin{array}{c}\text { Cream } \\
\text { Machine }\end{array}$ & $\begin{array}{c}\text { Chur } \\
\text { n }\end{array}$ & $\begin{array}{l}\text { Milking } \\
\text { Machine } \\
\text { (Mobile) }\end{array}$ & $\begin{array}{l}\text { Milking } \\
\text { Facility } \\
\end{array}$ & $\begin{array}{c}\text { Incubato } \\
\mathbf{r}\end{array}$ & $\begin{array}{c}\text { Traile } \\
\mathbf{r}\end{array}$ & $\begin{array}{c}\text { Ladl } \\
\mathrm{e}\end{array}$ & $\begin{array}{c}\text { Feed Preparation } \\
\text { Machine }\end{array}$ & $\begin{array}{c}\text { Water } \\
\text { Tank }\end{array}$ & $\begin{array}{c}\text { Pum } \\
\text { p }\end{array}$ \\
\hline $\begin{array}{l}2011- \\
2012\end{array}$ & 0.60 & 2.38 & 62.99 & 0.00 & 0.00 & 1.07 & 1.70 & 0.00 & 17.39 & 0.00 \\
\hline $\begin{array}{l}2012- \\
2013 \\
\end{array}$ & -0.20 & -1.36 & 28.38 & 33.33 & 0.00 & 1.58 & 0.00 & 1.80 & 7.41 & 1.33 \\
\hline $\begin{array}{l}2013- \\
2014 \\
\end{array}$ & -0.32 & -6.62 & 21.26 & 32.50 & 0.00 & 0.69 & 1.68 & -1.77 & 3.45 & $\begin{array}{c}- \\
1.32 \\
\end{array}$ \\
\hline $\begin{array}{l}2014- \\
2015 \\
\end{array}$ & 0.57 & 1.72 & 2.66 & 0.00 & 300.00 & 4.26 & $\begin{array}{c}15.9 \\
3 \\
\end{array}$ & -4.50 & 6.67 & $\begin{array}{c}- \\
1.33 \\
\end{array}$ \\
\hline $\begin{array}{l}2015- \\
2016\end{array}$ & 0.16 & 0.59 & 0.96 & -9.43 & 41.67 & 0.69 & 0.95 & 0.94 & 0.00 & 1.35 \\
\hline $\begin{array}{l}\text { 2016- } \\
2017\end{array}$ & -0.13 & 0.09 & 78.08 & 10.42 & 29.41 & 5.19 & 6.70 & 5.61 & 153.13 & 0.00 \\
\hline $\begin{array}{l}2017- \\
2018\end{array}$ & 0.84 & -0.62 & 0.84 & 0.00 & 0.00 & 0.09 & 0.00 & 0.00 & 0.00 & 0.00 \\
\hline $\begin{array}{l}2018- \\
2019\end{array}$ & 0.44 & 0.39 & 1.66 & 5.66 & 18.18 & 2.80 & $\begin{array}{c}49.3 \\
3\end{array}$ & 4.42 & -27.16 & 8.00 \\
\hline $\begin{array}{l}2019- \\
2020 \\
\end{array}$ & -9.51 & 4.15 & 6.75 & 266.07 & -7.69 & 3.20 & 1.50 & 4.24 & 0.00 & 3.70 \\
\hline$\%$ & -0.84 & \begin{tabular}{|l|}
0.08 \\
\end{tabular} & 22.62 & 37.62 & 42.40 & 2.18 & 8.43 & 1.19 & 17.88 & 1.30 \\
\hline $\begin{array}{l}\text { Projecti } \\
\text { on }\end{array}$ & $\begin{array}{c}\text { Cream } \\
\text { Machine }\end{array}$ & $\begin{array}{c}\text { Chur } \\
\text { n }\end{array}$ & $\begin{array}{l}\text { Milking } \\
\text { Machine } \\
\text { (Mobile) }\end{array}$ & $\begin{array}{l}\text { Milking } \\
\text { Facility }\end{array}$ & $\begin{array}{c}\text { Incubat } \\
\text { or }\end{array}$ & $\begin{array}{c}\text { Trail } \\
\text { er }\end{array}$ & $\begin{array}{c}\text { Lad } \\
\text { le }\end{array}$ & $\begin{array}{c}\text { Feed Preparation } \\
\text { Machine }\end{array}$ & $\begin{array}{l}\text { Water } \\
\text { Tank }\end{array}$ & $\begin{array}{c}\text { Pum } \\
\text { p }\end{array}$ \\
\hline 2021 & 9290 & 6757 & 1766 & 282 & 34 & 5801 & 366 & 124 & 70 & 170 \\
\hline 2022 & 9212 & 6811 & 2165 & 388 & 49 & 5927 & 397 & 126 & 82 & 172 \\
\hline 2023 & 9135 & 6866 & 2655 & 534 & 69 & 6056 & 431 & 127 & 97 & 175 \\
\hline 2024 & 9058 & 6921 & 3255 & 735 & 99 & 6188 & 467 & 129 & 114 & 177 \\
\hline 2025 & 8982 & 6976 & 3992 & 1012 & 141 & 6323 & 507 & 130 & 134 & 179 \\
\hline 2026 & 8906 & 7032 & 4895 & 1393 & 200 & 6461 & 549 & 132 & 158 & 182 \\
\hline 2027 & 8831 & 7088 & 6002 & 1917 & 285 & 6602 & 596 & 134 & 187 & 184 \\
\hline 2028 & 8757 & 7145 & 7360 & 2638 & 406 & 6746 & 646 & 135 & 220 & 186 \\
\hline 2029 & 8684 & 7202 & 9024 & 3630 & 578 & 6893 & 700 & 137 & 259 & 189 \\
\hline 2030 & 8611 & 7260 & 11066 & 4995 & 823 & 7043 & 759 & 138 & 306 & 191 \\
\hline
\end{tabular}

\section{Conclusions and Recommendations}

Since mechanization is a high-cost production input, it needs to be chosen and applied correctly, otherwise it can negatively affect the profitability in enterprises. Since the most economical use of this input can only be possible by planning models that are suitable for local conditions, increasing the efficiency of mechanization in agriculture can be achieved by making agricultural mechanization planning correctly (Demir, 2013).

Based on the calculated projection coefficient, it is anticipated that the greatest increase in soil tillage machines for the 2021 to 2030 period will be observed in cultivators by $38.40 \%$, corresponding to 10572 units, and the greatest reduction will be in rollers by -12.61 , corresponding to 18 units; the greatest increase in sowing, planting and fertilization machines will be observed in solid manure spreaders by $37.16 \%$ corresponding to 377 units; the greatest increase in plant protection machines will be observed in stretcher, motorized sprayer duster combined atomizer by $6.79 \%$ corresponding to 23 units, and the greatest decrease will be observed in motorised sprayers by -0.13 corresponding to 9 units; the greatest increase in harvesters will be observed in potato harvesting machines by $20.37 \%$ corresponding to 26 units, and the greatest reduction will be observed in reaper machines by -8.89 corresponding to 20 units; the greatest increase in other machinery will be observed in incubator machines by $42.40 \%$ corresponding to 823 units, and the greatest decrease will be observed in crèam machines by -0.84 corresponding to 8611 .

As a result of the positive projection coefficients determined for the 29 machines examined in the research, it was concluded that the technology usage projection in the agriculture of Ardahan province will increase until 2030, and there will be a decrease in these machines in line with the negative projection coefficient determined for 6 machines. Since grain production, animal husbandry and dairy products have an important place in Ardahan province, it has been concluded that machines in this class tend to increase and that supporting the producers within the scope of these machines can bring a positive trend to the provincial agriculture.

\section{References}

Altay, F., Turhal, K., 2011. The Current Situations of Agricultural Mechanization in Bilecik and Solution Proposals. 6th International Advanced Technologies Symposium (IATS'11), 16-18 May, Elazığ, Turkey. (In Turkish) 
Anonymous, 2021a. Turkey Republic Ardahan Governorship, Provincial Directorate of Environment and Urbanization. Ardahan Province Environmental Status Report for 2017. https://webdosya.csb.gov.tr/db/ced/icerikler/ardahan_2017_ cdr_son-20180605095733.pdf (Date of Access: 12/06/2021). (In Turkish)

Anonymous, 2021b. Turkish Statistical Institute, Crop Production Statistics 2021 https://tuikweb.tuik.gov.tr/PreTable.do?alt _id=1001 (Date of Access: 22/03/2021). (In Turkish)

Anonymous, 2021c. Turkish Statistical Institute, Agricultural Equipment and Machinery Statistics. https://biruni.tuik.gov.tr/medas/?kn=92\&locale=tr $\quad$ (Date of Access: 10/06/2021). (In Turkish)

Anonymous, 2021d. Ardahan Province Cultivated Agricultural Fields Product Report for 2020. Turkish Statistical Institute. (In Turkish)

Baran, M. F, Gökdoğan, O, Eren, Ö, Bayhan, Y., 2019, Projection of Technology Equipment Usage in Agricultural in Turkey, Türk Tarım ve Doğa Bilimleri Dergisi, 6(1): 1-9.

Bayram, M., Altuntaş, E., 2016. Investigation of Agricultural Mechanization Characteristics of Tokat Province for 2003 and 2013 Years. Tarım Makinaları Bilimi Dergisi, 12(3): 213-220. (In Turkish)

Demir, B., 2013. Projection of Technology Usage in Agriculture of Mersin Province. Alınteri Zirai Bilimler Dergisi, 24(B), 29-34. (In Turkish)

Demir, B., Kuş, E., 2016. Projection of Technology Using in Agriculture of Central Anatolia Region. Nevşehir Bilim ve Teknoloji Dergisi TARGiD Özel Say1, 89-95. (In Turkish)

Kaya, Y., Arısoy, R.Z., Taner, A., Aksoyak, Ş., Partigöç, F., Gültekin, İ., 2010. Comparison of Conventional Plating and Direct Seeding for Wheat Cheak Pea Rotations in Central Anatolian in Rainfed Conditions. Tarım Makinaları Bilimi Dergisi, 6(4): 267-272. (In Turkish)

Koçtürk, D., Onurbaş Avcıŏlu, A., 2007. Determining Agricultural Mechanization Level in Terms of thr Regions and the Cities in Turkey. Tarım Makinaları Bilimi Dergisi, 3(1): 17-24. (In Turkish)

Saral, A., Vatandaş, M., Güner, M., Ceylan, M., Yenice, T., 2000. Mechanization Situation of Turkish Agriculture" Union of Chamber of Engineers and Architects of Turkey Chamber of Agriculture 5th Technical Congress, 17-21 January, 901923, Ankara. (In Turkish)

Solak, M., Saltuk, B., Baran, M.F., 2019. Projection of Agricultural Tools and Machınery Usage in Agriculture in Siirt. Ispec Uluslararası Tarım ve Kırsal Kalkınma Kongresi, 10-12 Haziran, Siirt, 71-79. (In Turkish) 